\title{
Cystic lesions of the orbit -An analytical study
}

\author{
Venkatesh Perumal $^{1 *}$, Menaka ${ }^{2}$, Shankar $^{3}$ \\ 1,2 Assistant Professor, ${ }^{3}$ Associate Professor, Dept. of Ophthalmology, ${ }^{\mathbf{1} 2}$ Government Mohan Kumaramangalam Medical College Hospital, \\ Salem, Tamil Nadu, ${ }^{3}$ Vinayaka Mission's Kirupananda Variyar Medical College and Hospitals, Salem, Tamil Nadu, India
}

\section{Article Info}

Received: $1^{\text {st }}$ February, 2019

Accepted: $26^{\text {th }}$ March, 2019

Published Online: $9^{\text {th }}$ September, 2019

Keywords: Dermoid cyst, Excision, Mucocele, Orbital cystic lesion, Parasitic cyst.

\begin{abstract}
Introduction: The various orbital lesions range from a cyst which might be due to trauma, vascular or congenital, to tumors which are of benign or malignant cause, which are considered as a challenging problem commonly faced by ophthalmologist.

Aim: To study the prevalence, etiopathogenesis and the treatment modalities for the various cystic lesions of the orbit.

Materials and Methods: A prospective longitudinal study was conducted for a period of one year between June 2015 and May 2016 in the department of ophthalmology of Government Mohan Kumaramangalam Medical College Salem. A total of 130 patients with the sign of proptosis were reported during that said period. Out of the 130 patients 37 patients who are diagnosed to be having cystic lesions were taken up for study analysis. Cystic lesions which were confined to conjunctiva, eyelid and intraocular structures were excluded, but lesions involving the anterior compartment along with conjunctiva involvement were included in the study. A semi-structured questionnaire was prepared to collect the history related to demographic details, history regarding the onset, duration, progression and the past history. It was followed by a complete general and ocular examination including visual acuity, examination of anterior and posterior segments, examination of the mass, hertelsexophthalmometry, field charting, colour vision, refraction, IOP measurement and forced duction test.

Results: Among the various type of cystic lesions reported in our study subjects parasitic lesions were found to be the most common type which was present in more than $30 \%$ of the patients and it was followed by dermoid cyst in $24.3 \%$ of the patients and the other lesions reported were mucocele $(21.6 \%)$, hemagioma (10.8\%) and lymphangioma (8.1\%). Only one patients had a cancerous lesion namely adenoid cystic carcinoma. The mean age for the most of the cystic lesions like parasitic, dermoid cyst, vascular lesions like hemangioma and lymphangioma was found to be less than 15 years, whereas for mucocele and adenoid cystic carcinoma the mean age was found to be more than 30 years.

Conclusion: Every orbital lesion which comes into notice because of symptoms should be evaluated thoroughly to rule out malignant potential and also to assess the mass effect in it. As most of the lesions occur in children, it is of utmost importance to intervene early as it
\end{abstract}

orbital lesions. ${ }^{2}$ The commonly occurring orbital cystic lesions were orbital cystic lesions were 1. Cavernous hemagioma, which commonly occurs in the age group between 20 and 50 years and is characterized by proptosis, difficulty in ocular movements and has a slow progressive nature. It is a benign vascular neoplasm of congenital etiology which is most commonly diagnosed by MRI. ${ }^{3} 2$. Lymphangioma, it is a condition which occurs in younger individuals with a slow progressive nature presenting with proptosis and severe pain and severe cases might damage the optic nerve and it is most commonly diagnosed by CT scan imaging. ${ }^{4}$ 3. Inflammation and abscess: acute periorbital cellulites which usually occurs due to infection of eyelid commonly presents with proptosis, scleral accurately discern the number, size, nature and extent of the

*Corresponding Author: Venkatesh Perumal, Assistant Professor, Dept. of Ophthalmology, Government Mohan Kumaramangalam Medical College Hospital, Salem, Tamil Nadu, India

Email: shnkr_radhakrishnan@yahoo.com

http://doi.org/10.18231/j.ijceo.2019.079 
thickning and subperiosteal abscess which might even lead onto complications like cerebritis, cerebral abscess, cavernous sinus thrombosis and blindness and this condition can be easily picked up by CT scan. ${ }^{5}$ 4. Pseudotumour, usually presents in the age group between 10 and 40 years as unilateral exophthalmos. Pain, proptosis and restricted eye movement are the presenting features of pseudotumor which is diagnosed with $\mathrm{CT}$ and later confirmed by biopsy. ${ }^{6,7}$ 5. Dermoid cyst, it is a benign congenital choriostoma of eye accounting for more than $40 \%$ of childhood orbital neoplasm, which commonly presents as a painless nodule located in the frontozygomatic suture or less commonly in frontoethmoidal or frontomaxillary sutures. ${ }^{8,9}$ 6. Orbital cysticercosis, of late merging as the kost common benign cystic lesions of the orbit which is caused by the larval form of the parasitic tapeworm Tanenia solium. It is clinically presented as subconjunctival cyst with prominent proptosis and restricted orbital motility cyst involving the optic nerve is rare and it is diagnosed with CT or MRI. ${ }^{9} 7$. Retinoblastoma, it is the most common intraocular malignancy of childhood with a high incidence of 1 in 15,000-30,000 live births. ${ }^{10}$ It usually presents as bilateral with features of leucokoria, strabismus, glaucoma or vision loss. ${ }^{11} 8$. Rhabdomyosarcoma, is the most common primary malignant orbital tumor in children arising from mesenchymal elements, which manifest as rapidly progressive exophthalmos over days to weeks. 9. Ocular melanoma, melanoma occurs both as benign and malignant form among the adults. Both benign and malignant melamomas occur more commonly in the older people and are rare in the pediatric population. 10. Malignant melanoma, arising from the uveal tract is the most common in adults, particularly among whites. 11. Lymphoma, it occurs from $6-8 \%$ of orbital tumors with a slight female predominance. The reported frequency of involvement is: conjunctiva, $20 \%$ to $33 \%$; orbit, $46 \%$ to $74 \%$; and eyelid, $5 \%$ to $20 \%$ and is diagnosed by PET $-\mathrm{CT} .{ }^{10,11}$

With the advent of modern diagnostic methods like ultrasonography, CT scan and MRI scans, it has become easy to differentiate cystic lesions from others. They play a superior role because of their safety, non invasiveness, easy procedures and accurate diagnosis, which are essential for better outcome of treatment. Therefore, a comprehensive study about the cystic lesions of the orbit had been undertaken.

\section{Aim}

To study the prevalence, etiopathogenesis and the treatment modalities for the various cystic lesions of the orbit.

\section{Materials and Methods}

A prospective longitudinal study was conducted for a period of one year between June 2015 and May 2016 in the department of ophthalmology of Government Mohan Kumaramangalam Medical College Salem. The study was started after getting clearance from the institutional ethical committee and the informed consent was obtained from all the study subjects involved in the study. All patients irrespective of age and gender presented with proptosis were included for the study. A total of 130 patients with the sign of proptosis were reported during that said period. Among those patients proptosis due to trauma, secondaries due to malignancies and thyroid related orbitopathy were excluded for our analysis. Of the 130 patients .37 patients who are diagnosed to be having cystic lesions were taken up for study analysis. Cystic lesions which were confined to conjunctiva, eyelid and intraocular structures were excluded, but lesions involving the anterior compartment along with conjunctiva involvement were included in the study. A semi-structured questionnaire was prepared to collect the history related to demographic details, history regarding the onset, duration, progression and the past history. It was followed by a complete general and ocular examination including visual acuity, examination of anterior and posterior segments, examination of the mass, hertelsexophthalmometry, field charting, colour vision, refraction, IOP measurement and forced duction test. All the cases were evaluated in Oculoplastic, cornea and external disease and when needed in retina clinic over a period of four years. A detailed history taking including systemic illnesses too was done; history taking was followed by a detailed ocular examination. A provisional clinical diagnosis was made in all the cases. To further support the clinical diagnosis various investigations like computed tomography (CT) scan, magnetic resonance imaging (MRI), X-Ray, blood investigations, thyroid hormone levels tests etc were done. After doing needed investigations a final clinical diagnosis was made and was entered in the proforma made for the study.

Post making the clinical diagnosis all study subjects were taken up for surgery which included either excision and biopsy or incisional biopsy. The surgically resected specimens fixed in the $10 \%$ formalin were sent to the department of Pathology for histopathological diagnosis. In the department of Pathology, thorough gross examination of each mass for its size, shape, and consistency was done. Several representative areas of tissue were taken from received surgical specimen \& subjected to routine paraffin embedding. Four to five sections $2-3 \mathrm{~mm}$ thick were taken from different areas of specimen \& processed in automatic tissue processor. Blocks were prepared with the help of leuckhart's piece. After trimming of blocks, sections 5-7 um thick were cut with help of automated microtome. Sections were floated on water at temperature of 45 degree \& were taken on aluminized slides. The sections were stained by haematoxylin \& eosin stain in all cases. Special stain such as PAS stain was used whenever required. A histopathological diagnosis thus made available was entered in the proforma. Patient care and further management was planned accordingly. All positive and relevant findings of study subjects were entered in a specially designed proforma and analyzed using statistical package for social sciences (SPSS) data version 21 and the statistical inference was derived using appropriate statistical tests. 


\section{Results}

Among the total 130 patients who had reported with proptosis 37 of them had cystic lesions and so the prevalence of cystic lesions among patients with proptosis is $28.4 \%$. The age wise distribution of the patients with cystic lesions shows that majority were in the age group of less than 20 years with a mean age of 12.8 years and the gender wise distribution shows that the male: female ratio was almost 2:1 (Table 2). Involvement of eyes were almost equal in number with $51.3 \%$ of the patients had right eye lesion and $48.7 \%$ had lesions in their left eye. Majority of the study subjects with the cystic lesions had eccentric (62.6\%) type of globe displacement and $37.4 \%$ had axial type of globe displacement (Table 3). Among the various type of cystic lesions reported in our study subjects parasitic lesions were found to be the most common type which was present in more than $30 \%$ of the patients and it was followed by dermoid cyst in $24.3 \%$ of the patients and the other lesions reported were mucocele $(21.6 \%)$, hemagioma
(10.8\%) and lymphangioma (8.1\%). Only one patients had a cancerous lesion namely adenoid cystic carcinoma (Table 4). The mean age for the most of the cystic lesions like parasitic, dermoid cyst, vascular lesions like hemangioma and lymphangioma was found to be less than 15 years, whereas for mucocele and adenoid cystic carcinoma the mean age was found to be more than 30 years and this difference in the age was found to be statistically significant $(\mathrm{p}<.05)$ (Table 5). In our study we found dermoid cyst to be more common among females than males and similarly hemangioma and lymphangioma was more common in males than females and the difference was found to be statistically significant, whereas all the other cystic lesions did not show a statistical significant difference between gender (Table 6). Treatment modalities for all the cystic lesions were excision and for hemagioma and lymphangioma periodic observation were made to see for any recurrence.

Table 1: Age and gender wise distribution of the cystic lesions among the study subjects

\begin{tabular}{|l|c|c|c|}
\hline \multicolumn{1}{|c|}{ Age group (in years) } & Male & Female & Total \\
\hline$<10$ & $8(32 \%)$ & $6(50 \%)$ & $14(37.8 \%)$ \\
\hline $10-20$ & $12(48 \%)$ & $5(41.6 \%)$ & $17(45.9 \%)$ \\
\hline$>20$ & $5(20 \%)$ & $1(8.3 \%)$ & $6(16.2 \%)$ \\
\hline Total & $25(100 \%)$ & $12(100 \%)$ & $37(100 \%)$ \\
\hline Mean \pm SD & $12.3 \pm 4.2$ & $13.4 \pm 3.8$ & $12.8 \pm 3.4$ \\
\hline
\end{tabular}

Table 2: Distribution of the study subjects based on the displacement of globe

\begin{tabular}{|c|c|c|}
\hline Types & Number of cases & Percentage \\
\hline Axial & 14 & 37.48 \\
\hline Eccentric & 23 & 62.16 \\
\hline
\end{tabular}

Table 3: Distribution of the cystic lesions confirmed by histopathological report among the study subjects

\begin{tabular}{|c|c|c|}
\hline Cystic lesions & Cases & Percentage \\
\hline Parasitic & 12 & 32.43 \\
\hline Dermoid & 9 & 24.32 \\
\hline Mucocele & 8 & 21.62 \\
\hline Haemangioma & 4 & 10.81 \\
\hline Lymphangioma & 3 & 8.11 \\
\hline Adenoid cystic carcinoma & 1 & 2.70 \\
\hline
\end{tabular}

Table 4: Mean and SD of the age of the patients with various cystic lesions

\begin{tabular}{|c|c|c|c|}
\hline Cystic lesions & Mean age & SD & \multirow{2}{*}{$<.0001$} \\
\hline Parasitic & 14.7 & 4.8 & \\
\hline Dermoid & 14.0 & 5.2 & \\
\hline Haemangioma & 13.8 & 5.8 & \\
\hline Lymphangioma & 7.33 & 3.5 & \multirow{1}{}{} \\
\hline Adenoid cystic carcinoma & 35 & 0 & \\
\hline Mucocele & 39.2 & 6.1 & \\
\hline
\end{tabular}

p- value derived by applying one or two way ANOVA 
Table 5: Gender wise distribution of the cystic lesions among the study subjects

\begin{tabular}{|c|c|c|c|c|}
\hline Cystic lesions & Male & Female & Total & P value \\
\hline Parasitic & $8(32 \%)$ & $4(33.3 \%)$ & $12(32.4 \%)$ & 0.715 \\
\hline Dermoid & $5(20 \%)$ & $4(33.3 \%)$ & $9(24.3 \%)$ & 0.0281 \\
\hline Mucocele & $5(20 \%)$ & $3(25 \%)$ & $8(21.6 \%)$ & 0.319 \\
\hline Haemangioma & $3(12 \%)$ & $1(8.3 \%)$ & $4(10.8 \%)$ & 0.0419 \\
\hline Lymphangioma & $3(12 \%)$ & 0 & $3(8.1 \%)$ & 0.0273 \\
\hline Adenoid cystic carcinoma & $1(4 \%)$ & 0 & $1(2.7 \%)$ & 0.185 \\
\hline Total & $25(100 \%)$ & $12(100 \%)$ & $37(100 \%)$ & \\
\hline
\end{tabular}

$\mathrm{P}$ value derived by applying Chi-square test

Table 6: Distribution of the study subjects based on the treatment modalities for the various cystic lesions

\begin{tabular}{|c|c|c|}
\hline Diagnosis & Number of cases & Treatment \\
\hline Parasitic & 12 & Excision \\
\hline Dermoid & 9 & Excision \\
\hline Mucocele & 8 & Excision \\
\hline Haemangioma & 2 & Excision \\
& 2 & Periodic observation \\
\hline Lymphangioma & 1 & Excision \\
& 2 & Periodic observation \\
\hline Adenoid cystic carcinoma & 1 & Excision \\
\hline
\end{tabular}

\section{Discussion}

Orbit is one such location in the body where a vast number of lesions can occur. Of the many lesions that affect the orbit, cystic lesions contribute a significant number. These have diverse clinical findings, histopathological features and pathogenesis. Lesions present most commonly with proptosis. In the present study we found $28.5 \%$ of the patients presented with proptosis had cystic lesions in their orbit as we excluded proptosis due to trauma, secondaries to malignancies and thyroid related orbitopathy. According to a study done by Gunalp et al showed that the histopathologic and clinical records on 128 orbital cystic lesions diagnosed during a 32-year period from 1963 to 1995, among which dermoid cysts was found to be the most frequent (38 cases, 29.7\%) followed by hydatid cysts (33 cases, 25.8\%), mucoceles (31 cases, 24.2\%), pyoceles (10 cases, 7.8\%), meningoencephaloceles (9 cases, 7.0\%), epidermal inclusion cysts ( 4 cases, $3.1 \%$ ), hematoceles (2 cases, $1.6 \%$ ) and teratoma (one case, $0.8 \%$ ). ${ }^{13,14}$ It also showed that $64.1 \%$ of patients with cystic lesions were aged 18 years or less and this study was found to be almost similar to the present study. A similar type of study done by Kaan Gunduz et al done on biopsy proven orbital lesions performed on 1092 cases in Turkey showed that secondary tumors was the most common accounting for nearly $50 \%$ of orbital lesion followed by cystic lesions, pseudotumors and vascular lesions with $12 \%, 10 \%$ and $6 \%$ respectively. Other less common orbital lesions reported in his study was gliomas and mesenchymal tumors accounting for 3\%, $1 \%$ had metastatic tumor and $0.3 \%$ had primary orbital melanoma. The conclusion of their study had shown that cavernous hemangioma and lymphoid tumors are the most common benign and malignant orbital tumor among adults, whereas among children it was dermoid and rhabdomyosarcoma. ${ }^{14}$ In our study we found parasitic lesion was the most common cystic lesion followed by dermoid cyst among children and mucocele adenoid cystic carcinoma was found to be more common among the adults.

Hydatidosis of orbit is a common ocular lesion among children and young adults presenting with unilateral, progressive proptosis along with ocular pain and defective vision. A proper history helps to have a clinical suspicion towards that disease. Very few patients shows positive for serology, whereas CT imaging is the diagnostic tool for detecting orbital cysticercosis. When orbital cysticercosis is detected it is mandate to evaluate other systems for cysticercosis especially liver and brain. Early intervention is necessary in cases of orbital cysticercosis either in the form of surgical excision or cryoextraction as quoted by Fuad Sami Haddad. In the present study we found parasitic cyst as the most common cystic lesions of orbit among the pediatric patients. ${ }^{15}$

A study done by Jerry A Shield on the orbital cystic lesions had highlighted that among children dermoid cyst is the most common form of orbital cysts and nearly $90 \%$ of the biopsy specimen after surgical removal from a child was reported as dermoid cyst. Further in his study he showed that the second most common congenital cystic lesion was neural cyst which is presented in the form of cephalocele or optic nerve meningocele. He also quoted mucocele in children as the most common secondary cyst which is commonly associated with cystic fibrosis. In his results he mentioned that the inflammatory lesions due to parasitic infestations leading onto hydatidoasis is very common among tropical areas, India being a tropical country we found hydatidosis as the most common orbital cyst in our study. He further emphasized that adenoid cystic carcinoma, rhabdomyosarcoma, lymphangioma are rare cystic lesions of the orbit which almost reflects the results of our study. Jerry A Shield suggested that computed tomography along 
with magnetic resonance imaging helps in differentiating cystic lesions which would help in the management planning. As his suggestion in our study also we performed CT scan for all the patients which helped us to arrive at a diagnosis and which were further confirmed by histopathological examination. Management of orbital cyst depends on the location and type of cyst ranging between excision and observation. As the previous studies quoted evaluating an orbital cyst should be based on clinical, radiological and histopathological features and all these results should be correlated for a better evaluation of an orbital cystss. ${ }^{16}$ Similarly in our study also we followed the clinical, radiological and histopathological criteria for diagnosing the cystic lesions of the orbit and it was managed by surgical excision followed by periodic observation.

\section{Conclusion}

Every orbital lesion which comes into notice because of symptoms should be evaluated thoroughly to rule out malignant potential and also to assess the mass effect in it. As most of the lesions occur in children, it is of utmost importance to intervene early as it may be vision threatening. All lesions should be evaluated radiologically and confirmed with histopathological examination. Those which cannot be diagnosed even with radiological investigation, excision and HPE can be used as the primary modality of diagnosis and treatment. In our study among children cysticercosis followed by dermoid was the most common lesion and in adults it was mucocele and adenocarcinoma. As the present study was done only on a limited number of samples we can't generalize the results and so more number of studies with a larger sample has to be conducted to comment on the commonest orbital cystic lesions.

\section{Source of Funding: None.}

\section{Conflict of Interest: None.}

\section{References}

1. Forrest AW. Intraorbitaltumors. Arch Ophthalmol. 1949;41:198-232.
2. Henderson JW, Farrow GM. Orbital tumors. 2nd edn. New York: Brian C Decker (Thieme-Stratton); 1980:67-74.

3. Atlas SW. The Orbit, Cra Com Tomo and MRI 1987;3:126

4. Atlas SW. The Orbit, Cra Com Tomo and MRI 1987;3:128.

5. Zimmerman RA. Bilanuik LT. CT of orbital infection and its cerebral complication. Am J Radiol. 1980;134:45-50.

6. Bernardino ME, Zimmerman RD, Citrin CM, Davis DO. Scleral Thicknening: A sing of orbital pseudotumor. Am J Roentgenol. 1977;129:703-6.

7. Dresner. Computed tomography of orbital myositis. Am J Radiol. 1984:143:671-4.

8. Disorders of the orbit. Kanski J. 2000;14:572.

9. Maurya RP, Patne S UC, Singh VP, Singh MK, Ritika, Dwivedi M, Srivastava T. Presentation pattern and management Outcome of ocular and periocular dermoid cyst. Int J Ocular Oncol Oculopalast. 2016;2(2):95-105.

10. Sekhar GC, Lemke BN. Orbital cysticercosis. Ophthalmol. 1997;104:1599.

11. Zimmerman RA, Bilanuik L.T. Littman P: Computed tomography of paediatric craniofascial sarcoma. CT: J Comput Tomogr. 1978;2:113-21.

12. Zimmerman RA, Bilanuik LT. Computed tomography in evaluation of patients with bilateral retinoblastomas. CT: $J$ Comput Tomogr. 1979;3:251-7.

13. Maurya RP, Singh VP, Bhushan P, Singh MK, Kumar M. Congenital orbital teratoma with bilateral anophthalmia: a rare presentation. World J Pathol. 2012;1: 35-38.

14. Disorders of the orbit. Kanski J. 2000;14:581.

15. Günalp I, Gündüz K. Cystic lesions of the orbit. Int Ophthalmol. 1996-1997;20(5):273-7.

16. lhan Günalp \& Kaan Günduz. Biopsy-proven orbital lesions in Turkey: A survey of 1092 cases over 30 years. Orbit. 1994;13(2)67-79. DOI: 10.3109/01676839409071466.

17. Mallajosyula, Subrahmanyam \& Mehbub Ul Kadir, Syeed \& Sami Haddad, Fuad \& Ramavath, Sucharitha. (2014). Hydatidosis of the Orbit. 10.1007/978-3-642-54359-3_23.

18. Jerry A Shields, Carol L Shields. Orbital cysts of childhoodclassification, clinical features, and management. Surv Ophthalmol. 2004;49(3):281-99.

How to cite this article: Perumal V, Menaka, Shankar. Cystic lesions of the orbit -An analytical study. Indian J Clin Exp Ophthalmol 2019;5(3):330-4. 